\title{
Can we rehabilitate everyone?
}

\section{Heywood rehabilitation ward for the most severely disabled psychiatric patients}

\author{
Tim Atkin, Research Assistant, Psychology Department, Prestwich Hospital; \\ Philip Meats, Consultant Psychiatrist, King's Mill Hospital, Mansfield Road, \\ Sutton-in-Ashfield NG 17 4JL; and Caroline Sincock, Top-Grade Clinical \\ Psychologist, Psychology Department, Prestwich Hospital, Bury New Road, \\ Manchester M25 7BL
}

There is an increasing momentum for the provision of care for people with chronic mental illness to be made in settings other than mental hospitals. One concern arising from this shift in emphasis is with those patients who may find it particularly difficult to live in the community.

At Prestwich Hospital, a survey was carried out within the rehabilitation services of those patients who, for a variety of reasons, were unable to be resettled under the regional resettlement initiatives. This identified four different groups of patients: those who were actively resistant to resettlement and relocation; those who have been difficult to manage in the past, are floridly disturbed and have behavioural excesses; those displaying positive behaviour disturbance problems, such as frequent verbal or physical aggression and sexually and socially unacceptable behaviour; and a group of highly dependent and disturbed patients who have been in hospital for many years and have severe mental health problems.

Heywood ward was set up to cater for the needs of this last group. In providing care, the emphasis was placed on promoting appropriate individualised care in an enabling friendly atmosphere. This was intended to encourage the individual to develop everyday living skills while promoting a greater degree of independence. The unit was set up with a high staff resident ratio - for the 12 patients, 5 whole time equivalent nursing assistants and 5.5 whole time equivalent qualified nurses were provided (although staff turnover and one RMN taking maternity leave reduced this at times). The multidisciplinary team included one full time OT helper and one half time clinical psychologist. Medical support included (weekly) three hours associate specialist time, five hours senior registrar time, one GP session and consultant cover. A pleasant ward environment was provided (with decorations, carpets, pot-plants, etc.) and all care staff were selected because of a particular interest in working with this group of patients.
We initially made as detailed as possible a clinical assessment of the client group and subsequently made repeated assessments at regular intervals, concentrating on level of functioning. Our aim in this paper is to describe the client group and their progress in the first 12 months, and then discuss the implications.

\section{The study}

Each individual's functioning was assessed by means of four measures. The first of these was the REHAB (Hall \& Baker, 1983), which comprised 23 items examining the level of functioning of the individual. The scoring was divided into two scales - deviant and general behaviour, the latter being further divided into subscales of self care, social activity, speech skills, speech disturbance and community skills. Each assessment was completed by a member of staff after a one week observation period.

The Social Behaviour Scale (SBS) (Wykes \& Sturt, 1986) was also used to assess functioning, but this time on the basis of the individual's behaviour over the previous month, assessed by means of an interview with a staff member. There were 23 items examining problems in all areas of functioning, and these yielded two scores - the total number of problem behaviours, severe and mild (BSM), and the total number of problem behaviours identified as severe (BSS).

Direct observation was also carried out, using a time sampling schedule drawn from Alevizos et al (1977). This involved three days of 12-hour long observation, each individual being observed once every hour for 30 seconds. The individual's activities are recorded as observed and later coded into various activities, such as verbal interaction, group activity, bizarre behaviour or no activity.

The other measure used was the Psychiatric Assessment Scale (PAS) (Krawiecka, Goldberg \& Vaughan, 1977). This was a checklist of eight 
symptoms, assessed by a psychiatrist during a short interview with the patient. As well as a total psychopathology rating, the measure provided subscales of affective, negative and positive symptomatology. Inter rater reliabilities were assessed for all these measures, and ranged from $74 \%$ for the REHAB to $91 \%$ for the Direct Observation schedule. The PAS was found to be $77 \%$ reliable and the SBS $89 \%$.

The 12 residents of Heywood Ward were extremely dependent on staff for personal hygiene and other self care needs. They were severely emotionally withdrawn and uncommunicative, and any changes in routine disturbed them. According to Research Diagnostic Criteria (Spitzer et al, 1978), most of the patients had chronic residual schizophrenia, while some were actively psychotic. Their average age was 70 years; their average length of stay in Prestwich Hospital was 34 years.

\section{Findings}

To illustrate the nature of the client group and show progress made, we give two representative case vignettes before describing the results of the repeated measures.

$\mathrm{Mr} \mathrm{A}$ is 70 years old. He was discharged from the army in 1940 to a psychiatric hospital and transferred to Prestwich in 1941. He described hearing voices of women which he thought were transmitted by wireless. He was solitary and uncommunicative, often talking and swearing to himself and over the years became almost mute. He avoided eye contact, laughed and smiled inappropriately, and exhibited bizarre stereotypic touching. On moving to Heywood ward he showed no conversational speech. There was occasional echolalic repetition of what had just been said to him or he muttered or swore to himself. The stereotypic touching was almost continuous. He was dependent on staff for supervision, dressing, washing and finding his way outside of the ward setting. Over the course of the year, he has slowly begun to interact more with staff, making brief eye contact and occasionally smiling. He usually gives a spoken response, but still does not initiate conversation. He appears more relaxed and less agitated. He leaves the ward on his own to go to a patients' cafe within the hospital grounds. He satisfies Research Diagnostic Criteria for chronic undifferentiated schizophrenia.

$\mathrm{Mr}$ B is 59 years old and has been in hospital for 37 years. After leaving school at the age of 14 , he worked in a cotton mill until he became ill at the age of 16 . His pre-morbid personality was described as shy and quiet, with few interests and little social life, but affectionate within the family. He became distressed and easily agitated, hearing voices, and was deluded that he was being persecuted by Germans. He spent time standing in front of a mirror, swearing, grimacing and stereotypically rubbing his hands, but he was mostly apathetic, not caring to wash or shave. He spent most of the day lying on a couch. On admission to hospital he continued to be apathetic and did not engage in any organised activity or help with self care or ward chores. He occasionally and briefly became overactive, singing loudly and smearing faeces. He made little if any response to neuroleptics, ECT or lithium. He had almost no spontaneous speech apart from importuning for cigarettes with the phrase "Gorrafag?". Apart from the singing, his only other verbal utterances were echolalic repetition of the last few words spoken to him. He appeared to understand spoken language as he was able to follow simple instructions. He was incontinent of urine and faeces and was a compulsive water drinker, even out of unflushed toilet bowls.

$\mathrm{Mr} \mathrm{B}$ has been encouraged to communicate and to improve his self care. Over the year he has slowly improved and will now attempt to wash his hands and face with simple verbal prompts. His communication has improved: he will spontaneously ask for cigarettes or drinks in the patients' cafe, or he will put his coat on and stand near a member of staff as a non-verbal prompt to be taken out. His episodes of agitation are less frequent and he is only occasionally drinking excessively from taps.

The data from the REHAB assessment indicated that the subjects were all considerably disabled across a wide range of areas-social activity, self care, communication and community skills - putting them at the most disabled end of the psychiatric hospital population.

The PAS results showed that the level of pathology present within the group did not change over the year of observation. There were consistently high levels of negative symptoms recorded, along with low levels of positive and affective symptoms. However, the PAS almost certainly underestimates the presence of hallucinations and delusions in this group, because they are so uncommunicative that these symptoms cannot be rated.

The SBS assessments showed a low level of functioning, as also suggested by the REHAB. There was a reduction in the number of severe behavioural problems encountered in caring for the residents. No particular area of functioning could be identified as producing this change; rather, all the residents improved in very different respects. The direct observation of residents showed a reduction in the time spent engaged in strange or inappropriate activities (from an average of $38 \%$ of daytime hours to $19 \%$ ).

These changes were borne out by informal reports, which agreed that the functioning of all the residents had improved in different respects. Despite the changes detected, it must be emphasised that the general level of functioning of these patients did not change. The levels of symptoms and disability within the group remained extremely high. From the REHAB norms, this client group remains at the most disabled end of the psychiatric hospital population.

\section{Comment}

This group of patients are among the most dependent and disabled in the hospital and would be incapable 
of living in the community at their present level of functioning. They have received considerable investment of time and of material resources, and have made worthwhile gains in skills and reduction of psychopathology over a year. It must be emphasised that these gains are of a different order of magnitude than those occurring on rehabilitation wards with higher functioning patients. An experienced member of staff, on moving from one such ward to Heywood ward, commented "You learn to appreciate it when somebody says one word, whereas before you just took it for granted". Thus any gains are small but important.

These gains may be related to the stability of the ward, with its infrequent changes in staff and no moving of patients to facilitate ward closure plans. There was no control group available, which is an obvious weakness of this study. Nevertheless, we feel that the improvements are genuine and reflect the increased efforts made by the staff and the particular atmosphere created on the ward.

Despite these improvements, these patients remain extremely disabled and dependent on nursing staff. The changes were not detected by the PAS or the REHAB, which continue to reflect severe psychopathology. It is therefore unlikely that, even with continued high staff input, these patients will ever be rehabilitated to the point of independence from staff. They still require more effort, for example, in prompting washing or dressing than if staff actually did the tasks for them. In this context, rehabilitation must be seen as attempting to facilitate gradual improvements in functioning without the pressure to achieve independence from this level of support. The improvements in these patients' quality of life are likely to be lost if they are returned to the level of care provided before they moved to Heywood Ward. Could this care be provided in the community? Whatever the setting, this group of patients would still need the same level of clinical commitment and resources.

\section{Conclusion}

This study provides evidence that worthwhile gains in level of functioning and in reduction of psychopathology can be achieved in those patients most disabled by chronic schizophrenia, through the provision of a suitable environment and intensive nursing care. Thus, to answer the title question, the principles of good rehabilitation practice can be used with benefit for even the most severely disabled psychiatric patients, but this does not mean that they will be restored to normal functioning. The improvements obtained by the residents of Heywood are certainly important, but are not sufficient to allow withdrawal of the extra investment made in them. Good quality care continues to demand increased resources.

\section{Acknowledgement}

We are most grateful for the continued assistance of the staff of Heywood ward, who have devoted much of their time and energy to this research.

\section{References}

Alevizos, P., DeRisi, W., Liberman, R., Eckman, T. \& Callahan, E. (1978) The Behaviour Observation Instrument: a method of direct observation for programme evaluation. Journal of Applied Behaviour Analysis, 11, 243-257.

HAll, J. \& BAKER, R. (1983) Rehabilitation Evaluation. Aberdeen: Vine Publishing.

Krawiecka, M., Goldberg, D. \& Vaughan, M. (1977) A standardised psychiatric assessment scale for rating chronic psychotic patients. Acta Psychiatrica Scandinavia, 55, 299-308.

SPITZER, R. L., ENDICOTT, J. \& Robins, E. (1978) Research Diagnostic Criteria: Rationale and Reliability. Archives of General Psychiatry, 35, 773-782.

WYKES, T. \& STURT, E. (1986) The measurement of social behaviour in psychiatric patients: an assessment of the reliability and validity of the SBS schedule. British Journal of Psychiatry, 148, 1-11. 